\title{
ASSOCIAÇÃO ENTRE OSTEOARTRITE DE JOELHO E SÍNDROME METABÓLICA: UMA REVISÃO SISTEMÁTICA
}

\author{
Association between knee osteoarthritis and metabolic syndrome: a systematic \\ review
}

\section{Asociación entre osteoartritis de rodilla y síndrome metabólico: una revisión sistemática}

\author{
Maura Fernandes Franco iD \\ Universidade Estadual de Campinas - UNICAMP - Campinas (SP) - Brasil
}

Daniel Vicentini de Oliveira (iD

Universidade Estadual de Campinas - UNICAMP - Campinas (SP) - Brasil

\author{
Arlete Maria Valente Coimbra (iD \\ Universidade Estadual de Campinas - UNICAMP - Campinas (SP) - Brasil
}

\section{RESUMO}

Objetivo: Identificar as possíveis associações entre osteoartrite de joelho (OA) e síndrome metabólica (SM). Métodos: Revisão sistemática de literatura, ocorrida entre os anos de 2012 e 2017, nas bases: Biblioteca Virtual em Saúde (BVS), SciELO, Cochrane e Embase; em português e/ou inglês, com os descritores "osteoartrite" e "síndrome metabólica" em ambas as línguas. Para avaliação da qualidade metodológica, utilizou-se a Declaração STROBE (Strengthening the Reporting of Observational Studies in Epidemiology). Selecionaram-se sete artigos, todos com qualidade metodológica alta. Resultados: Os dados apontam para associação entre OA sintomática e hipertensão arterial sistêmica, dislipidemia e o número de fatores acumulados, com aumento da gravidade e progressão dos sintomas em mulheres. Entretanto, ainda não se esclareceu por completo a associação entre o estado pré-inflamatório da SM e a OA. Conclusão: Os resultados encontrados sugerem que uma melhor compreensão das ações das vias metabólicas envolvidas com a osteoartrite de joelho poderia abrir uma nova avenida terapêutica a esses pacientes.

Descritores: Osteoartrite; Síndrome Metabólica; Obesidade; Hipertensão.

\section{ABSTRACT}

Objective: To identify possible associations between knee osteoarthritis (KO) and metabolic syndrome (MS). Methods: $A$ systematic review was carried out between 2012 and 2017 on the Virtual Health Library (VHL), SciELO, Cochrane and Embase databases to search for publications in Portuguese and/or English using the descriptors "osteoarthritis" and "metabolic syndrome" in both languages. Methodological quality was assessed using the STROBE (Strengthening the Reporting of Observational Studies in Epidemiology) Statement. Seven articles with high methodological quality were selected. Results: The data point to the association between symptomatic $\mathrm{KO}$ and systemic arterial hypertension, dyslipidemia and number of accumulated factors, with increased severity and progression of symptoms in women. However, the association between the pre-inflammatory stage of MS and KO has not yet been fully clarified. Conclusion: The findings suggest that a better understanding of the actions of the metabolic pathways involved in KO could open a new avenue for the treatment of these patients.

Descriptors: Osteoarthritis; Metabolic Syndrome; Obesity; Hypertension.

\section{RESUMEN}

Objetivo: Identificar las posibles asociaciones entre la osteoartritis de rodilla (OA) y el síndrome metabólico (SM). Métodos: Revisión sistemática de la literatura entre los años de 2012 y 2017 en las bases de datos Biblioteca Virtual de Salud (BVS), SciELO, Cochrane y Embase; en portugués y/o inglés con los descriptores "osteoartritis" y "síndrome metabólico" en ambos los idiomas. Para la evaluación de la calidad metodológica se utilizó la Declaración STROBE (Strengthening the Reporting of

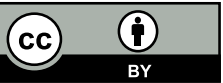


Observational Studies in Epidemiology). Se han seleccionado siete artículos y todos con alta calidad metodológica. Resultados: Los datos señalan la asociación entre la OA sintomática y la hipertensión arterial sistémica, la dislipidemia y el número de factores acumulados, con el aumento de la severidad y la progresión de los síntomas en mujeres. Sin embargo, todavía no se ha aclarado por completo la asociación entre el estado pre-inflamatorio del SM y la AO. Conclusión: Los resultados encontrados sugieren que una mejor comprensión de las acciones de las vías metabólicas de la osteoartritis de rodilla puede ser una nueva opción terapéutica para eses pacientes.

Descriptores: Osteoartritis; Síndrome Metabólico; Obesidad; Hipertensión.

\section{INTRODUÇÃO}

A osteoartrite $(\mathrm{OA})$ é uma condição musculoesquelética que afeta milhões de pessoas e representa a primeira causa da deficiência no mundo após os 40 anos ${ }^{(1-3)}$. É considerada a maior causa de incapacidade física e apresenta-se pela dor, rigidez e limitações nas atividades de vida diária $(A V D)^{(3-5)}$. Sua incidência aumenta com o avanço da idade: cerca de $40 \%$ dos adultos com idade acima de 70 anos apresentam OA de joelhos, e $80 \%$ dos que apresentam a doença têm algum tipo de limitação de movimento(5).

Embora tenha sido, historicamente, vista como uma doença do tipo desgaste, ela é atualmente considerada uma doença inflamatória de baixo grau, resultante da perda do equilíbrio entre degradação e reparação celular dentro da cartilagem por meio dos condrócitos ${ }^{(6)}$. Sua patogênese e progressão parecem ser resultado da interação complexa e dinâmica de mecânica, fatores moleculares celulares e sistêmicos ${ }^{(7-9)}$.

Devido à sua heterogeneidade, subdivide-se em subgrupos fenotípicos ${ }^{(9)}$ que, apesar de apresentarem diferenças causais importantes, provavelmente compartilham elementos em comum, como envelhecimento, fatores biomecânicos e alterações metabólicas ${ }^{(9,10)}$.

Durante o século 20, a síndrome metabólica (SM) aumentou drasticamente devido às mudanças no estilo de vida da população (aumento da ingestão de calorias e comportamento sedentário) ${ }^{(10)}$. A Organização Mundial da Saúde (OMS) ${ }^{(11)}$ definiu a SM como uma alteração do metabolismo da glicose e/ou resistência insulínica associada à hipertensão arterial sistêmica (HAS), ao aumento de triglicérides do plasma e/ou à diminuição do colesterol High Density Lipoproteins (HDL) e obesidade central ou abdominal; no entanto ainda não há uma definição única. Acomete principalmente pessoas acima 45 anos, está presente em 10 a 30\% da população mundial, é prevalente em 59\% dos pacientes com OA ${ }^{(1)}$, e os indivíduos com OA e SM, concomitantemente, apresentam sintomas mais graves e precoces que a população em geral ${ }^{(2)}$.

A associação entre a diabetes mellitus (DM) e OA foi descrita pela primeira vez em 1961 ${ }^{(12)}$, porém o primeiro grande estudo que explorou essa associação foi realizado apenas em $2007^{(13)}$. Os autores demonstraram que pacientes com DM tipo 2 apresentaram taxas duas vezes maiores de artroplastia de joelho ou quadril decorrente de OA que os sujeitos não diabéticos. Também observaram que os pacientes diabéticos apresentaram maiores níveis de inflamação sinovial e dor ${ }^{(13)}$.

A partir de então, propôs-se um novo subtipo de OA, a OA metabólica ${ }^{(14)}$. O envolvimento dos fatores metabólicos na etiologia da OA é apoiado por estudos epidemiológicos, bem como por dados experimentais ${ }^{(15)}$. Tanto os achados in vivo quanto in vitro têm demonstrado que os mediadores inflamatórios derivados do tecido adiposo, hiperglicemia, dislipidemia e da inflamação crônica de baixo grau, têm efeito prejudicial direto sobre o metabolismo da cartilagem ${ }^{(1-3,10,15)}$.

Visto o risco de comprometimento funcional da OA, o risco cardiovascular e as elevadas taxas de morbimortalidade da SM são encarados, atualmente, como obstáculos à qualidade de vida e uma importante questão de saúde pública ${ }^{(8-10)}$. Mudança nos hábitos de vida e a prática regular de exercício físico, dentre outras atitudes saudáveis, levam à prevenção do surgimento de novos sintomas, ao retardo do agravamento dos sintomas já existentes e à redução do risco para a saúde ${ }^{(16,17)}$. Assim, o controle dos sinais e sintomas da OA e da SM são tidos como essenciais na promoção de uma vida saudável(18).

Compreender os mecanismos pelos quais os fatores metabólicos e a OA de joelho se associam e agridem o organismo humano é fundamental para o desenvolvimento e direcionamento de terapias mais específicas e eficazes na prevenção dos sintomas e promoção da saúde. Assim, o objetivo desta revisão é identificar as possíveis associações entre osteoartrite de joelho e síndrome metabólica. 


\section{MÉTODOS}

A presente investigação embasou-se em uma revisão sistemática de literatura de trabalhos indexados nas bases de dados eletrônicos de livre acesso Biblioteca Virtual em Saúde (BVS), Scielo, Cochrane e Embase. Com o intuito de selecionar os artigos mais recentes e respeitando o cronograma original da pesquisa, analisaram-se estudos publicados entre janeiro de 2012 e abril de 2017.

Realizou-se uma busca por artigos que possuíam como descritores os termos osteoartrite (osteoarthritis) e síndrome metabólica (metabolic syndrome), utilizando o conector "e" ('and'). Como critérios de inclusão, adotaram-se: trabalhos publicados na íntegra, em inglês e/ou português, e que haviam sido realizados com seres humanos. Como critérios de exclusão, consideraram-se: a) artigos que eram editoriais, carta ao leitor e/ou revisão de literatura; b) que não investigavam a associação entre OA e SM; c) que não abordavam a OA isolada de joelho.

Durante a primeira etapa da investigação, fez-se um levantamento dos artigos encontrados, com os descritores propostos, nas bases de dados anteriormente citadas. Em uma segunda etapa aplicaram-se os critérios de exclusão pré-estabelecidos e, em uma terceira etapa, realizou-se a análise dos títulos e dos resumos dos estudos, além da exclusão das duplitas e daqueles que não satisfaziam os critérios de inclusão. Em um quarto momento, realizouse uma leitura criteriosa dos artigos completos e a análise qualitativa dos mesmos. O processo de seleção, leitura e análise dos artigos foi conduzido por pares. Nos casos de desacordo entre os revisores, houve discussão entre eles a fim de definir se haveria a integração daquele estudo à pesquisa.

A qualidade metodológica dos estudos incluídos deu-se através do Strengthening the Reporting of Observational Studies in Epidemiology ou Declaração STROBE ${ }^{(19)}$. Trata-se de uma lista de verificação, com 22 itens, com recomendações sobre o que deve ser incluído em uma descrição mais precisa e completa de estudos. Esses itens são codificados em zero (item não contemplado) ou um (contemplado), indicando, portanto, o nível de qualidade do estudo. Quanto maior a pontuação, melhor a qualidade do artigo. Utilizou-se esta estratégia a fim de identificar a qualidade dos estudos: de maior qualidade (acima de $70 \%$ do escore), média qualidade (entre 50 a $69 \%$ ) e baixa qualidade (abaixo de $50 \%$ ).

\section{RESULTADOS}

Após análise detalhada dos títulos e resumos, sete artigos mostraram-se pertinentes à revisão: Niu et al.,

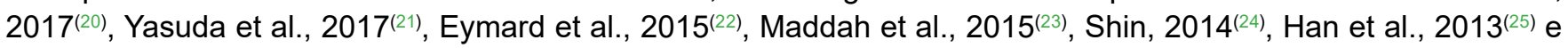
Jungmann et al., 2013 ${ }^{(26)}$.

A Figura 1 evidencia as etapas do processo de seleção dos estudos e o número de estudos identificados, selecionados, excluídos e incluídos na revisão.

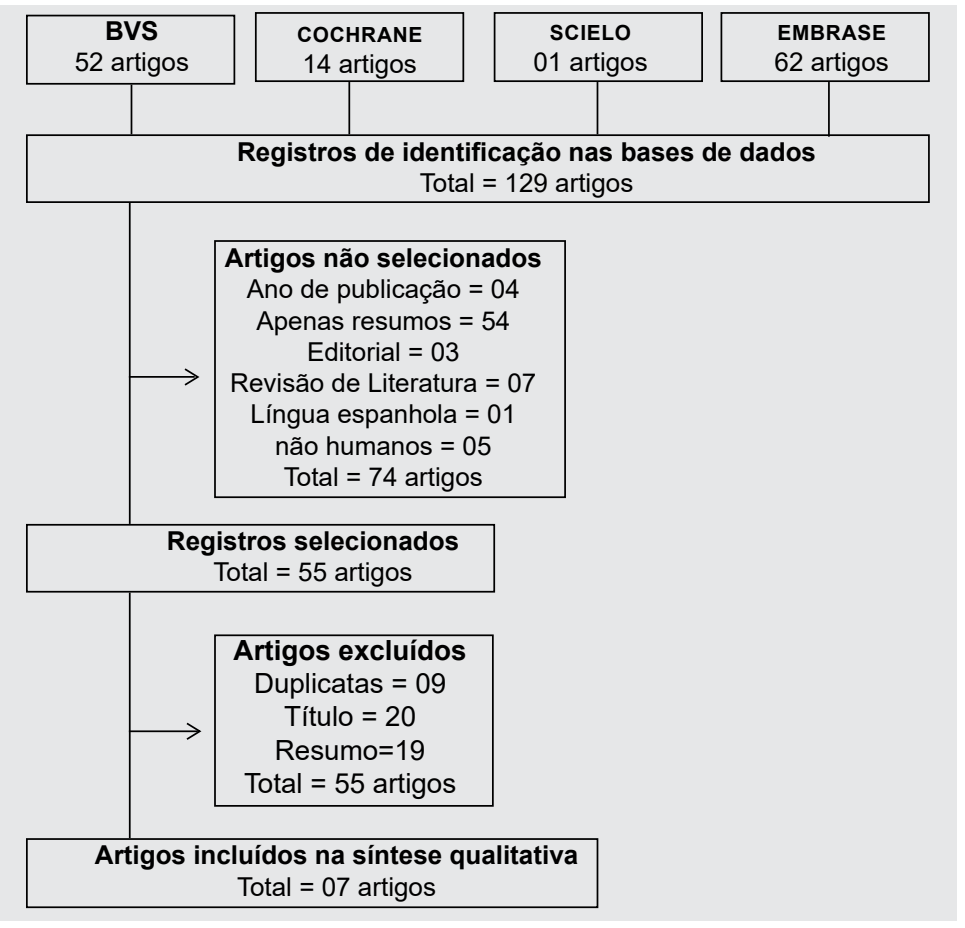

Figura 1 - Fluxograma das etapas de seleção dos estudos para revisão sistemática. 
A revisão sistemática compôs-se pela análise dos sete artigos que investigaram a associação entre a OA de joelho e a SM. Detalhes sobre composição da amostra, local da pesquisa, critério diagnóstico da OA e critério diagnóstico da SM em cada artigo estão apresentados no Quadro I.

Quadro I - Caracterização dos estudos analisados.

\begin{tabular}{|c|c|c|c|c|c|}
\hline Autor & Amostra & $\begin{array}{l}\text { Idade / } \\
\text { Média }\end{array}$ & $\begin{array}{l}\text { Estudo / } \\
\text { Local }\end{array}$ & OA & SM \\
\hline $\begin{array}{l}\text { Niu et al. } \\
2017^{(20)}\end{array}$ & 991 sujeitos & $\begin{array}{c}>40 \text { anos } \\
54.2\end{array}$ & $\begin{array}{c}\text { Framingham } \\
\text { EUA }\end{array}$ & $K L \geq 2$ & NCEP ATPIII \\
\hline $\begin{array}{c}\text { Yasuda et al. } \\
2017^{(21)}\end{array}$ & 119 mulheres & $\begin{array}{c}45-88 \text { anos } \\
69.0\end{array}$ & Japão & $K L \geq 2$ & $\begin{array}{c}\text { Examination Committee } \\
\text { of Criteria for Metabolic } \\
\text { Syndrome in Japan }\end{array}$ \\
\hline $\begin{array}{c}\text { Eymard et al. } \\
2015^{(22)}\end{array}$ & 559 pacientes & $\begin{array}{c}>50 \text { anos } \\
62.8\end{array}$ & $\begin{array}{c}\text { Estudo SEKOIA } \\
18 \text { países }\end{array}$ & $\begin{array}{c}\text { JSW } \\
\text { (medida } \\
\text { anual) }\end{array}$ & $\begin{array}{l}\text { NCEP ATPIII } \\
{ }^{*} \text { Autorrelato }\end{array}$ \\
\hline $\begin{array}{l}\text { Maddah et al. } \\
\qquad 2015^{(23)}\end{array}$ & 625 sujeitos & $\begin{array}{c}>18 \text { anos } \\
\text { Homem } 55.4 \\
\text { Mulher } 54.4\end{array}$ & Irã & $\mathrm{KL} \geq 2$ & NCEP ATPIII \\
\hline $\begin{array}{c}\text { Shin } \\
2014^{(24)}\end{array}$ & 2.363 sujeitos & $\begin{array}{c}\geq 50 \text { anos } \\
63.4\end{array}$ & $\begin{array}{c}\text { Estudo KNHANES } \\
\text { Coréia }\end{array}$ & $K L \geq 2$ & NCEP ATPIII \\
\hline $\begin{array}{l}\text { Han et al. } \\
2013^{(25)}\end{array}$ & 2.234 sujeitos & $\begin{array}{c}\text { 40- } 91 \text { anos } \\
\text { Com OA } 64.5 \\
\text { Sem OA } 53.2\end{array}$ & $\begin{array}{c}\text { Estudo KNHANES } \\
\text { Coréia }\end{array}$ & Autorrelato & NCEP ATPIII \\
\hline $\begin{array}{c}\text { Jungmann et al } \\
\qquad 2013^{(26)}\end{array}$ & 403 sujeitos & $\begin{array}{l}45-60 \text { anos } \\
\text { Homem } 52.0 \\
\text { Mulher } 52.2\end{array}$ & $\begin{array}{c}\text { The } \\
\text { Osteoarthritis/nitiative } \\
\text { Multicêntrico/ EUA }\end{array}$ & $K L \geq 2$ & $\begin{array}{l}\text { NCEP ATPIII } \\
{ }^{*} \text { Autorrelato }\end{array}$ \\
\hline
\end{tabular}

OA: Osteoartrite; SM: Síndrome Metabólica; EUA: Estados Unidos da América; KL: Kellgreen-Lawrence; SEKOIA: Strontium Ranelate in Knee Osteoarthritis; JSW: Joint Space Width; KNHANES: Korean National Health and Nutrition Examination Survey; NCEP ATP III: National Cholesterol Education Program-Adult Treatment Panel III

Todos os estudos são observacionais, sendo três longitudinais ${ }^{(2,22,26)}$ e quatro transversais ${ }^{(21,23-25)}$. Além disso, dois são multicêntricos ${ }^{(22,25)}$, seis realizaram-se com indivíduos de ambos os $\operatorname{sexos}^{(20,22-26)} \mathrm{e}$ um apenas com mulheres ${ }^{(21)}$.

Quanto ao ano de publicação, dois são de $2017^{(20,21)}$, dois são de $2015^{(22,23)}$, um é de $2014^{(24)}$ e dois são de $2013^{(25,26)}$. Nos anos de 2012 e 2016 não houve publicação que se enquadrasse nesta revisão.

Em relação à idade, um artigo utilizou indivíduos acima de $18 \operatorname{anos}^{(23)}$, dois utilizaram indivíduos acima de 40 $\operatorname{anos}^{(20,25)}$, dois utilizaram indivíduos acima de $45 \operatorname{anos}^{(21,26)}$ e dois utilizaram indivíduos acima de 50 anos ${ }^{(22,24)}$. Apenas um deles possuía limite de idade máxima ${ }^{(26)}$, estipulada em 60 anos, e nenhum deles desenvolveu-se exclusivamente com idosos. Em um artigo a média de idade dos indivíduos superou 65 anos $^{(21)}$.

Sobre a composição da amostra, um estudo investigou indivíduos internados para cirurgia eletiva de joelho ${ }^{(21)}$, dois avaliaram indivíduos ambulatoriais com OA sintomática ${ }^{(22,23)}$, quatro artigos abordaram indivíduos da comunidade ${ }^{(20,24-26)}$ e nenhum estudo investigou indivíduos institucionalizados.

Para a definição da SM, seis artigos embasaram-se no NCEP ATP III ${ }^{(20,22-26)}$, entretanto dois desses utilizaram o autorrelato para alguns dos fatores metabólicos ${ }^{(2,26)}$, como diabetes e triglicérides, e um estudo utilizou outro critério diagnóstico, o Examination Committee of Criteria for Metabolic Syndrome do Japão ${ }^{(21)}$.

Já para o diagnóstico da $O A$, cinco estudos utilizaram a escala de $K L \geq 2^{(20,12,23,24,26)}$, um utilizou a medida do espaço articular ${ }^{(22)}$ e um utilizou o autorrelato(25).

Todos os artigos obtiveram a pontuação STROBE ${ }^{(19)}$ entre 16 e 22, o que caracteriza um percentual de qualidade alto (entre $72,72 \%$ e $100 \%$ ). Os itens não pontuados relacionavam-se à falta da descrição dos elementos-chave do desenho do estudo; à descrição do contexto, lugares e datas relevantes; às medidas adotadas para evitar viés; à discriminação dos participantes em cada fase do estudo; à especificação dos fatores de confusão; ao resumo dos principais objetivos e das limitações do estudo na discussão; à possibilidade de generalização dos resultados e à falta de descrição de financiamento da pesquisa. 


\section{DISCUSSÃO}

Para $57 \%$ dos pesquisadores, a OA de joelho prevalece entre pessoas do sexo feminino e com idades mais avançadas $^{(20,23-25)}$. Para $29 \%$, as pessoas com OA apresentam maior índice de massa corporal (IMC) ${ }^{(20,23)}$, maior medida de CC, maiores valores de pressão arterial sistólica (PAS), maiores níveis de LDL $^{(23-25)}$, maior nível de tabagismo, menor peso, menor altura, menor consumo de álcool e menor nível de atividade física ${ }^{(20,25)}$. Já para $14 \%$ eles apresentam menor grau de escolaridade ${ }^{(20)}$.

Em relação à SM: $29 \%$ dos artigos relataram que sua prevalência é maior entre os homens e que cresce com o avanço da idade ${ }^{(23-25)} ; 14 \%$ mostraram que, entre as mulheres, a medida da CC associa-se a uma maior prevalência da $\mathrm{OA}^{(25)}$ e que o fato de ter SM aumenta em $49 \%$ a probabilidade da $\mathrm{OA}^{(24)} \mathrm{em}$ ambos os sexos.

Sobre os fatores metabólicos isolados: $29 \%$ dos estudos encontraram uma associação significativa entre a DM tipo 2 e a redução do espaço articular do joelho ${ }^{(22,26)}$. Porém $50 \%$ referem que tal associação se dá apenas em homens ${ }^{(22)}$ e, para outros $50 \%$, ela se dá em ambos os sexos ${ }^{(26)}$.

Os artigos demostraram que o acúmulo dos fatores metabólicos, independentemente do IMC, esteve associado a uma maior degradação da cartilagem articular em $14 \%$ das publicações ${ }^{(26)}$, e esses autores sugerem haver uma interferência metabólica negativa na composição bioquímica da cartilagem. Por outro lado, em $43 \%$ dos relatos não houve associações entre os componentes metabólicos ${ }^{(20-22)}$, sejam eles isolados, sejam acumulados, com OA radiográfica de joelho após os ajustes de confusão.

Tanto os fatores isolados quanto a SM estão associados à OA de joelho, mas não independentes do IMC e do peso corporal(20-22). Uma revisão sistemática recente sobre o assunto não encontrou tal associação(27). Nesta, os pesquisadores relataram que, após os ajustes para IMC ou peso, a associação entre a SM e a OA radiográfica de joelho não se manteve significativa nos estudos analisados.

Sobre SM e OA sintomática, observou-se uma associação significativa entre HAS, dislipidemia e número de fatores acumulados, com aumento da gravidade e progressão dos sintomas em mulheres em $14 \%$ das pesquisas ${ }^{(18)}$, mesmo após os ajustes para IMC e idade. No caso, os autores apontam que o acúmulo de componentes metabólicos está associado com a maior intensidade de dor, independentemente da idade, sexo e peso ${ }^{(18)}$.

Algumas divergências nos resultados das pesquisas possivelmente são decorrentes da heterogeneidade das amostras - idade, sexo e condições de saúde diferentes - e da falta de padronização (e até mesmo de algumas adaptações) dos critérios diagnósticos. Autores também encontraram dados conflitantes e insuficientes para estabelecer uma conclusão definitiva sobre tal associação. Além disso, sugerem que futuros estudos utilizem amostras mais robustas e que foquem nos estágios iniciais das doenças e nas manifestações de componentes metabólicos ao longo da vida ${ }^{(27)}$.

Incluir informações sobre dieta e exercícios saudáveis pode reduzir custos adicionais para o gerenciamento da sintomatologia da $O A^{(21,26)}$. Medidas preventivas e mudanças no estilo de vida vêm sendo destacados como provedores de saúde. A dieta mediterrânea apresenta-se associada não apenas a uma menor prevalência de $\mathrm{OA}$ de joelho ${ }^{(10,28)}$ e doença cardiovascular (DCV) ${ }^{(29)}$, mas também associada com melhor qualidade de vida, menores níveis de dor e deficiência funcional, e menor índice de depressão entre pacientes com AO de joelho ${ }^{(30)}$.

O monitoramento e a prevenção da SM por meio de ações de intervenção do setor saúde e dos programas específicos do Sistema Único de Saúde, direcionados para redução de fatores de risco, atuando no controle da obesidade, na prática de exercício físico regular, na melhora no perfil lipídico e glicêmico e no controle dos níveis pressóricos, devem ser considerados ${ }^{(31)}$.

É altamente benéfico educar e estimular a sociedade a se manter saudável, inclusive aqueles indivíduos sem a presença dos sintomas, isto é, de maneira preventiva. É importante destacar, entretanto, que a efetividade dos programas de incentivo à saúde depende, dentre outros fatores, de comportamentos contextuais e fatores situacionais, como níveis de dor, autoeficácia e consciência de recomendação ${ }^{(32)}$. Assim, os provedores e facilitadores desses programas devem considerar a saliência desses fatores para autogestão e promoção da saúde.

Estudos longitudinais, com critérios padronizados e que busquem avaliar o impacto específico dos componentes metabólicos e hiperglicêmicos crônicos na progressão de OA se mostram interessantes e devem ser incentivados.

\section{CONCLUSÃO}

Conclui-se que uma melhor compreensão das ações das vias metabólicas envolvidas no início e na progressão da OA poderá abrir uma nova avenida terapêutica, com foco no equilíbrio glicêmico e, juntamente com programas de promoção da saúde, promover uma vida mais saudável a esses pacientes. 


\section{CONFLITOS DE INTERESSE}

Esta pesquisa não apresenta conflitos de interesses.

\section{CONTRIBUIÇÕES}

Maura Fernandes Franco contribuiu com a elaboração e delineamento do estudo; aquisição, análise e interpretação de dados; redação e/ou revisão do manuscrito; Daniel Vicentini de Oliveira contribuiu com a análise e interpretação de dados; Arlete Maria Valente Coimbra contribuiu com a elaboração e delineamento do estudo; revisão do manuscrito.

\section{FONTES DE FINANCIAMENTO}

Esta pesquisa recebeu apoio financeiro da Coordenação de Aperfeiçoamento de Pessoal de Nível Superior (CAPES).

\section{REFERÊNCIAS}

1. Le Clanche S, Bonnefont-Rousselot D, Sari-Ali E, Rannou F, Borderie D. Inter-relations between osteoarthritis and metabolic syndrome: a common link?. Biochimie. 2016;121:238-52.

2. Courties A, Sellam J, Berenbaum F. Metabolic syndrome-associated osteoarthritis. Curr Opin Rheumatol. 2017;29(2):214-22.

3. Courties A, Sellam J. Osteoarthritis and type 2 diabetes mellitus: What are the links?. Diabetes Res Clin Pract. 2016;22:198-206.

4. Sellam J, Berenbaum F. Is osteoarthritis a metabolic disease? Joint Bone Spine. 2013;80(6):568-73.

5. Marques CDL, Duarte ALBP. A importância do reconhecimento de comorbidades em pacientes com osteoartrite. Temas Reumatol. 2011;12(1):3-6.

6. Sun AR, Friis T, Sekar S, Crawford R, Xiao Y, Prasadam I. Is synovial macrophage activation the inflammatory link between obesity and osteoarthritis?. Curr Rheumatol Rep. 2016;18(9):57.

7. Leardini G, Salaffi F, Caporali R, Canesi B, Rovati L, Montanelli L, et al. Direct and indirect costs of osteoarthritis of the knee. Clin Exp Rheumatol. 2004;22(6):699-706.

8. Nuesch E, Dieppe P, Reichenbach S, Williams S, Iff S, Jüni P. All cause and disease specific mortality in patients with knee or hip osteoarthritis: population based cohort study. BMJ. 2011;342:d1165.

9. Mobasheri A, Rayman MP, Gualillo O, Sellam J, van der Kraan P, Fearon U. The role of metabolism in the pathogenesis of osteoarthritis. Nat Rev Rheumatol. 2017;13(5):302-11.

10. Farnaghi S, Crawford R, Xiao Y, Prasadam I. Cholesterol metabolism in pathogenesis of osteoarthritis disease. Int J Rheum Dis. 2017;20(2):131-40.

11. Abella V, Scotece M, Conde J, López V, Lazzaro V, Pino J, et al. Adipokines, metabolic syndrome and rheumatic diseases. J Immunol Res. 2014;2014:343746.

12. Chung SM, Hyun MH, Lee E, Seo HS. Novel effects of sarcopenic osteoarthritis on metabolic syndrome, insulin resistance, osteoporosis, and bone fracture: the national survey. Osteoporos Int. 2016;27(8):2447-57.

13. Schett G, Kleyer A, Perricone C, Sahinbegovic E, lagnocco A, Zwerina J, et al. Diabetes is an independent predictor for severe osteoarthritis: results from a longitudinal cohort study. Diabetes Care. 2013;36(2):403-9.

14. Hawker GA, Stanaitis L. Osteoarthritis year in review 2014: clinical. Osteoarthr Cartil. 2014;22(12):1953-7.

15. Kluzek S, Newton JL, Arden NK. Is osteoarthritis a metabolic disorder?. Br Med Bull. 2015;115(1):111-21.

16. Renne I, Gobbens RJJ. Effects of frailty and chronic diseases on quality of life in Dutch community-dwelling older adults: a cross-sectional study. Clin Interv Aging. 2018;2018(13):325-34.

17. Katikireddi SV, Skivington K, Leyland AH, Hunt K, Mercer SW. The contribution of risk factors to socioeconomic inequalities in multimorbidity across the lifecourse: a longitudinal analysis of the Twenty- 07 cohort. BMC Med. 2017;15(1):152. 
18. Chi WC, Wolff J, Greer R, Dy S. Multimorbidity and decision-making preferences among older adults. Ann Fam Med. 2017;15(6):546-51.

19. Malta M, Cardoso LO, Bastos FI, Magnanini MMF, Silva CMFP. Iniciativa STROBE: subsídios para a comunicação de estudos observacionais. Rev Saúde Pública. 2010;44(3):559-65.

20. Niu J, Clancy M, Aliabadi P, Vasan R, Felson DT. Metabolic syndrome, its components, and knee osteoarthritis: The Framingham Osteoarthritis Study. Arthritis Rheumatol. 2017;69(6):1194-203.

21. Yasuda E, Nakamura R, Matsugi R, Goto S, Ikenaga Y, Kuroda K, et al. Association between the severity of symptomatic knee osteoarthritis and cumulative metabolic factors. Aging Clin Exp Res. 2018;30(5):481-8.

22. Eymard F, Parsons C, Edwards MH, Petit-Dop F, Reginster JY, Bruyère O, et al. Diabetes is a risk factor for knee osteoarthritis progression. Osteoarthr Cartil. 2015;23(6):851-9.

23. Maddah S, Mahdizadeh J. Association of metabolic syndrome and its components with knee osteoarthritis. Acta Med Iran. 2015;53(12):743-8.

24. Shin D. Association between metabolic syndrome, radiographic knee osteoarthritis, and intensity of knee pain: results of a national survey. J Clin Endocrinol Metab. 2014;99(9):3177-83.

25. Han CD, Yang IH, Lee WS, Park YJ, Park KK. Correlation between metabolic syndrome and knee osteoarthritis: data from the Korean National Health and Nutrition Examination Survey (KNHANES). BMC Public Health. 2013;13:603.

26. Jungmann PM, Kraus MS, Alizai H, Nardo L, Baum T, Nevitt MC, et al. Association of metabolic risk factors with cartilage degradation assessed by $\mathrm{T} 2$ relaxation time at the knee: data from the osteoarthritis initiative. Arthritis Care Res. 2013;65(12):1942-50.

27. Li S, Felson DT. What is the evidence to support the association between metabolic syndrome and osteoarthritis? - A systematic review. Arthritis Care Res. 2018.

28. Veronese N, Stubbs B, Noale M, Solmi M, Luchini C, Smith TO, et al. Adherence to a Mediterranean diet is associated with lower prevalence of osteoarthritis: data from the osteoarthritis initiative. Clin Nutr. 2017;36(6):1609-14.

29. Ros E, Martínez-González MA, Estruch R, Salas-Salvadó J, Fitó M, Martínez JA, et al. Mediterranean diet and cardiovascular health: teachings of the PREDIMED study. Adv Nutr. 2014;5(3):330S-6S.

30. Veronese N, Stubbs B, Noale M, Solmi M, Luchini C, Maggi S. Adherence to the Mediterranean diet is associated with better quality of life: data from the Osteoarthritis Initiative. Am J Clin Nutr. 2016;104:1403-09.

31. Rocha FL, Melo RLP, Menezes TN. Fatores associados à síndrome metabólica em idosos do interior do Nordeste brasileiro. Rev Bras Geriatr Gerontol. 2016;19(6):978-86.

32. Booker S. Predictors of engagement in recommended self-management behaviors for osteoarthritis pain. J. Pain. 2018;19(3):S30.

\section{Endereço para correspondência:}

Maura Fernandes Franco

Universidade Estadual de Campinas - UNICAMP

Rua Tessália Vieira de Camargo, 126

Bairro: Cidade Universitária Zeferino Vaz

CEP: 13083-887 - Campinas - SP - Brasil

E-mail: mauraffranco@gmail.com

Como citar: Franco MF, Oliveira DV, Coimbra AMV. Associação entre osteoartrite de joelho e síndrome metabólica: uma revisão sistemática. Rev Bras Promoç Saúde. 2019;32:8448. 